\title{
Effect of Microwaves on the Current-Phase Relation of Superconductor-Normal-Metal-Superconductor Josephson Junctions
}

\author{
M. Fuechsle, J. Bentner, D. A. Ryndyk, M. Reinwald, W. Wegscheider, and C. Strunk \\ Fakultät für Physik, Universität Regensburg, D-93040 Regensburg, Germany \\ (Received 27 July 2007; revised manuscript received 1 August 2008; published 23 March 2009)
}

\begin{abstract}
We investigate the current-phase relation (CPR) of long diffusive superconductor-normal-metalsuperconductor $(\mathrm{Nb} / \mathrm{Ag} / \mathrm{Nb})$ Josephson junctions in thermodynamic equilibrium and under microwave irradiation. While in equilibrium good agreement with the predictions of quasiclassical theory is found, we observe that the shape of the CPR can be strongly affected by microwave irradiation. Close to a Josephson-phase difference $\varphi \simeq \pi$, the supercurrent can be strongly suppressed when increasing the rf power. Our results can be understood in terms of microwave excitation of low-lying Andreev bound states across the minigap in the junction. In the frequency interval studied, this mechanism becomes important, when the minigap closes at $\varphi \simeq \pi$.
\end{abstract}

DOI: 10.1103/PhysRevLett.102.127001

PACS numbers: 74.25.Nf, 74.45.+c, 74.50.+r

The fundamental mechanism of Cooper-pair transfer across superconductor-normal-metal-superconductor (SNS) Josephson junctions relies on the existence of Andreev bound states (ABS) in the normal metal [1]. The energies $\epsilon$ of the ABS depend on the phase difference $\varphi$ between the two superconductors. Diffusive normal metals exhibit a continuous spectrum of ABS, described by a complex supercurrent spectral density $j_{\mathrm{S}}(\epsilon, \varphi) \quad[2,3]$. Assuming perfectly transparent interfaces at a distance $d$, $j_{\mathrm{S}}(\epsilon, \varphi)$ is determined by two parameters, namely, the energy gap $\Delta$ of the superconductor, and the Thouless energy $\epsilon_{\mathrm{th}}=\hbar D / d^{2}$, where $D$ is the diffusion constant. The ABS spectrum exhibits a minigap $\epsilon_{g}(\varphi)$, which closes as $\varphi$ approaches $\pi$. The total supercurrent is given by [2]

$$
I_{\mathrm{S}}(\varphi)=\frac{1}{e R_{\mathrm{N}}} \int_{0}^{\infty} \operatorname{Im} j_{\mathrm{S}}(\epsilon, \varphi)[1-2 f(\epsilon)] d \epsilon,
$$

where $R_{\mathrm{N}}$ is the resistance of the normal conductor and in thermal equilibrium $f(\epsilon)$ is the Fermi distribution function determining the occupation probability of the ABS at temperature $T$. As opposed to the case of conventional tunneling junctions, for ideal NS interfaces higher order scattering processes become important. These processes correspond to the phase-coherent transfer of multiple Cooper pairs across the junction, and manifest themselves in a nontrivial Fourier representation $I_{\mathrm{S}}(T, \varphi)=$ $\sum_{j} I_{\mathrm{C}}^{(j)}(T) \sin (j \varphi)$ of Eq. (1). In thermodynamic equilibrium, the higher harmonics $I_{\mathrm{C}}^{(j)}$ are rapidly suppressed for temperatures larger than $T_{\mathrm{th}}=\epsilon_{\mathrm{th}} / k_{B}$.

Attempts to detect higher harmonics in the CPR of diffusive SNS junctions by measuring the magnetic response of loops with an embedded junction date back to the 1970s [4], but failed because of the contradictory requirements of large $\epsilon_{\text {th }}$ (very low temperatures and short junctions) and low $I_{\mathrm{C}}$ (higher $T$ and long junctions) to avoid hysteretic switching of the loop [2]. As an alternative method, the detection of subharmonic Shapiro steps was suggested $[5,6]$. These experiments were successful, but resulted in a very surprising nonmonotonic $T$ dependence of the higher order coefficients $I_{\mathrm{C}}^{(j)}$. As opposed to the monotonic exponential suppression with $T$ expected from theory [3], the subharmonic Shapiro steps were observed most clearly at $T>T_{\text {th }}$. At the highest temperatures even $I_{\mathrm{C}}^{(1)}<I_{\mathrm{C}}^{(2)}$ and $I_{\mathrm{C}}^{(1)}<I_{\mathrm{C}}^{(3)}$ were found [6]. These findings have so far remained in contradiction with the equilibrium quasiclassical theory and were explained by the generation of nonequilibrium by the dc bias required to record the $I V$ characteristics.

In this Letter, we present a systematic experimental investigation of the current phase relation in long diffusive $\mathrm{Nb} / \mathrm{Ag} / \mathrm{Nb}$ junctions within and out of thermodynamic equilibrium. Empoying Waldram's method [4,7], we directly detect the supercurrent with a micro-Hall sensor [8]. Compared to previous experiments [5,6], our observations are not affected by additional dynamical effects induced by a dc voltage bias. The results demonstrate the possibility to control the occupation of Andreev levels by a microwave field, which opens important possibilities for the operation of Andreev-quantum bits [9].

To investigate the supercurrent response of our SNS junctions to an applied phase difference, we integrated the junctions into superconducting loops. The gauge invariant phase difference $\varphi$ across the junction is then related to the total magnetic flux $\Phi$ via $\varphi=-2 \pi \Phi / \Phi_{0}$, where $\Phi_{0}=h / 2 e$ is the magnetic flux quantum [10]. The samples were patterned on top of the active area of micronsized Hall crosses (see insets in Fig. 1) which have been structured into a GaAs/AlGaAs semiconductor heterostructure containing a 2-dimensional electron gas $190 \mathrm{~nm}$ below the surface. With a mean free path of $l=9 \mu \mathrm{m}$ at $4 \mathrm{~K}$ and an active area of $10 \times 10 \mu \mathrm{m}^{2}$ these Hall probes work close to the ballistic regime. Depending on the bath 


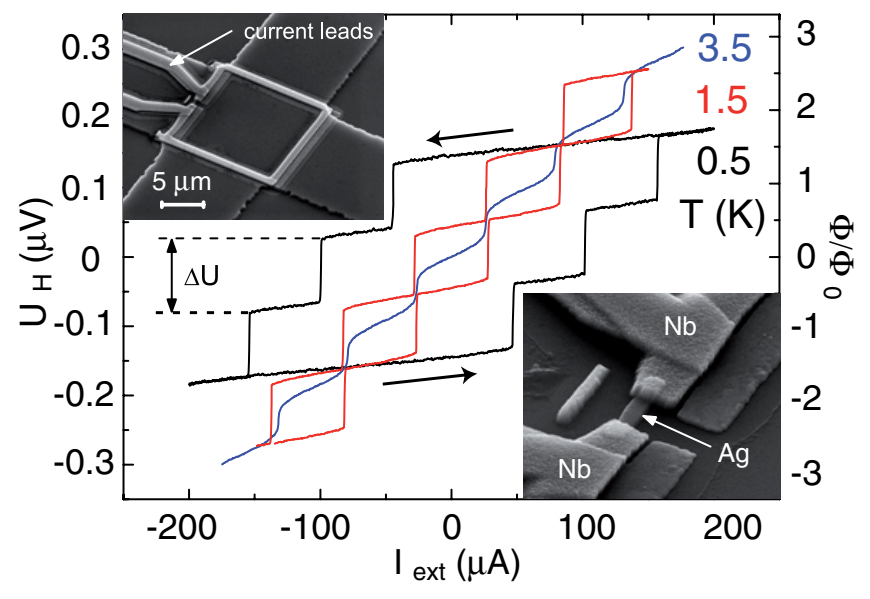

FIG. 1 (color online). The measured Hall voltage $U_{\mathrm{H}}$ as a function of the loop current $I_{\text {ext }}$. For low temperatures $(\beta>1)$ the effective flux shows hysteretic behavior; hence, only the rising branch of the CPR is accessible. Upper inset: SEM image of the $\mathrm{Nb}$ loop with an edge length of $10 \mu \mathrm{m}$ and an inductance $L \approx 40 \mathrm{pH}$ fabricated on the active area of a micro-Hall-cross. Lower inset: close-up of the actual SNS junction. The thickness of the $\mathrm{Ag}(\mathrm{Nb})$ layer is $40 \mathrm{~nm}(150 \mathrm{~nm})$. While the spacing between the $\mathrm{Nb}$ electrodes is $d=495 \mathrm{~nm}$, the effective length of the Ag bridge is $d_{\text {eff }}=640 \mathrm{~nm}$ with a width of $210 \mathrm{~nm}$.

temperature, which sets an upper limit to the applied current through the Hall probe, $I_{p}$, we obtain a supercurrent sensitivity between 0.1 and $2 \mu \mathrm{A}$, corresponding to a flux sensitivity of $10^{-3}$ to $2 \times 10^{-2} \Phi_{0}$. The fabrication process makes use of $e$-beam lithography and a shadow evaporation technique based on a thermostable trilayer mask system. We use a sacrificial layer of polyether sulfone (PES) as described in the work of Dubos et al. [11] and a mask layer of silicon nitride (SiN) [12]. The insets to Fig. 1 show scanning electron micrographs of the loop on top of the Hall cross, and a close-up of the $\mathrm{Nb} / \mathrm{Ag} / \mathrm{Nb}$ junction, respectively.

The magnetic flux is controlled by driving an external current $I_{\text {ext }}$ through the loop via current leads close to the SNS contact (Fig. 1, upper inset). This has the advantage that both the external flux $L I_{\mathrm{ext}}$ and the flux $L I_{\mathrm{S}}$ induced by the junction are detected with the same filling factor $\alpha$, which greatly facilitates the calibration of the measured Hall voltage $U_{\mathrm{H}}$ in terms of $\Phi_{0}$ :

$$
\Phi=\alpha \Phi_{\mathrm{H}}=\alpha^{\prime} U_{\mathrm{H}}=L\left(I_{\mathrm{ext}}-I_{\mathrm{S}}\left(2 \pi \Phi / \Phi_{0}\right)\right) .
$$

Here, $\Phi_{\mathrm{H}}$ is the flux enclosed by the active area $A$ of the Hall cross, $\alpha^{\prime}=\alpha$ Ane $/ I_{p}, e=1.602 \times 10^{-19}$ As, $n=$ $2.26 \times 10^{15} \mathrm{~m}^{-2}$, and $L$ is the geometric inductance of the loop. Depending on the match between the loop area and $A, \alpha$ values between 0.3 and 0.4 can be achieved, limited by the finite distance between the 2DEG and the loop.

Figure 1 shows the Hall voltage $U_{\mathrm{H}}\left(I_{\text {ext }}\right)$ of a sample with $d=495 \mathrm{~nm}$ for different temperatures. The magnetic response of the SNS loop is superimposed on a linear background caused by $I_{\text {ext }}$, the slope of which can be used to determine $L$. Below a certain temperature, when the parameter $\beta_{\mathrm{L}}=2 \pi L I_{\mathrm{C}}(T) / \Phi_{0}$ exceeds 1 , the response is hysteretic with distinct jumps when the critical current of the junction is exceeded. In the limit $\beta_{\mathrm{L}} \gg 1$, the step height corresponds to one flux quantum and can thus be used to determine $\alpha^{\prime}$. A plot of $I_{\mathrm{S}}=I_{\mathrm{ext}}-\alpha^{\prime} U_{\mathrm{H}} / L$ vs $\varphi=2 \pi \alpha^{\prime} U_{\mathrm{H}} / \Phi_{0}$ represents the desired CPR.

The Thouless energy $\epsilon_{\text {th }}$ of our junctions is determined by the effective length $d_{\text {eff }}>d$ of the normal conducting barrier, which accounts for the fact that the Andreev reflections at the SN interface occur randomly within the overlap length $s$ between $\mathrm{Nb}$ and $\mathrm{Ag}$. As in earlier work [13], we have chosen $d_{\text {eff }}=d+s$. The gap parameter $\Delta=1.3 \mathrm{meV}$ of our $\mathrm{Nb}$ films has been extracted from its measured $T_{c} \simeq 8.5 \mathrm{~K}$. Figure 2 shows the measured CPR for several temperatures below $1 \mathrm{~K}$. While at $1 \mathrm{~K}$ the CPR is still a perfect sine, at lower temperatures clear deviations from the sinusoidal shape are observed. The inset to Fig. 2 shows $I_{\mathrm{C}}(T)$, extracted from the maximum supercurrent [14], which agrees very well with the quasiclassical theory $[3,15]$ using $D=0.014 \mathrm{~m}^{2} / \mathrm{s}$ as the only fitting parameter (solid line in inset). This value of $D$ corresponds to a $\epsilon_{\mathrm{th}}=24 \mu \mathrm{eV}$ for this sample $\left(d_{\mathrm{eff}}=\right.$ $640 \mathrm{~nm}$ ). With all parameters fixed, we are now prepared for a quantitative comparison of the measured CPR with the theoretical predictions [3], represented by the solid lines in Fig. 2. Within our experimental resolution the agreement is excellent [16]. In particular, the result for our lowest accessible temperature of $300 \mathrm{mK}=1.1 \epsilon_{\mathrm{th}} / k_{B}$ almost coincides with the theoretical predictions for this

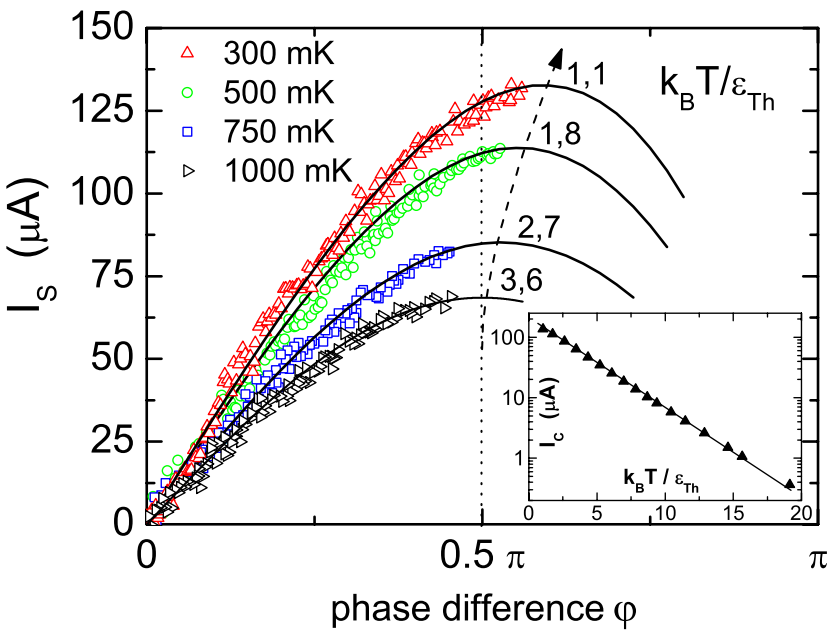

FIG. 2 (color online). CPR for low temperatures down to $k_{B} T \approx \epsilon_{\mathrm{th}}$. For $T \lesssim 500 \mathrm{mK}$ a clear deviation from the sinusoidal Josephson relation is observed. The solid lines correspond to the predictions of the quasiclassical theory, with all parameters determined independently. Inset: The critical current as a function of temperature. From the fit of the data to the quasiclassical theory (solid line) we determine the value $D=0.014 \mathrm{~m}^{2} / \mathrm{s}$ of the diffusion constant in the $\mathrm{Ag}$ bridge. 
value of $\Delta / \epsilon_{\mathrm{th}}=58$ in the limit $T \rightarrow 0$, where the degree of anharmonicity is largest.

We now turn to the main subject of the present Letterthe effect of microwave irradiation on the CPR in the frequency range between 3 and $12 \mathrm{GHz}$. The rf power is applied at room temperature and reduced by several attenuators at $4 \mathrm{~K}, 1 \mathrm{~K}$, and $100 \mathrm{mK}$ (in total $-60 \mathrm{~dB}$ including cable losses) to ensure proper thermalization of the rf cable, which is terminated with a small loop in the vicinity of the sample. Figure 3(a) shows the CPR measured at $2.85 \mathrm{~K}$ for different levels of the externally applied power $P$. These data are taken away from a cavity resonance of the sample chamber. At this temperature, the equilibrium CPR for $P=0$ is nearly sinusoidal and the critical current is small enough $\left(\beta_{\mathrm{L}}<1\right)$ to access the full CPR. It is clearly seen that with increasing power the maximum supercurrent is reached for lower $\varphi$ values, $\varphi_{\max }<\pi / 2$, in contrast to the behavior at low temperatures (see Fig. 2). The effect is most prominent for phase differences close to $\pi$, where a pronounced suppression of the supercurrent occurs. For $\varphi<\varphi_{\max }$, the supercurrent even appears to be slightly enhanced compared to $P=0$. At certain frequencies, which we attribute to resonances of our sample chamber, we found that $I_{\mathrm{C}}^{(1)}$ and $I_{\mathrm{C}}^{(2)}$ can even be comparable [see inset in Fig. 3(a)]. This can even lead to an inversion of the supercurrent direction close to $\varphi \lesssim \pi$. In Fig. 3(b) the temperature dependence of the effect is

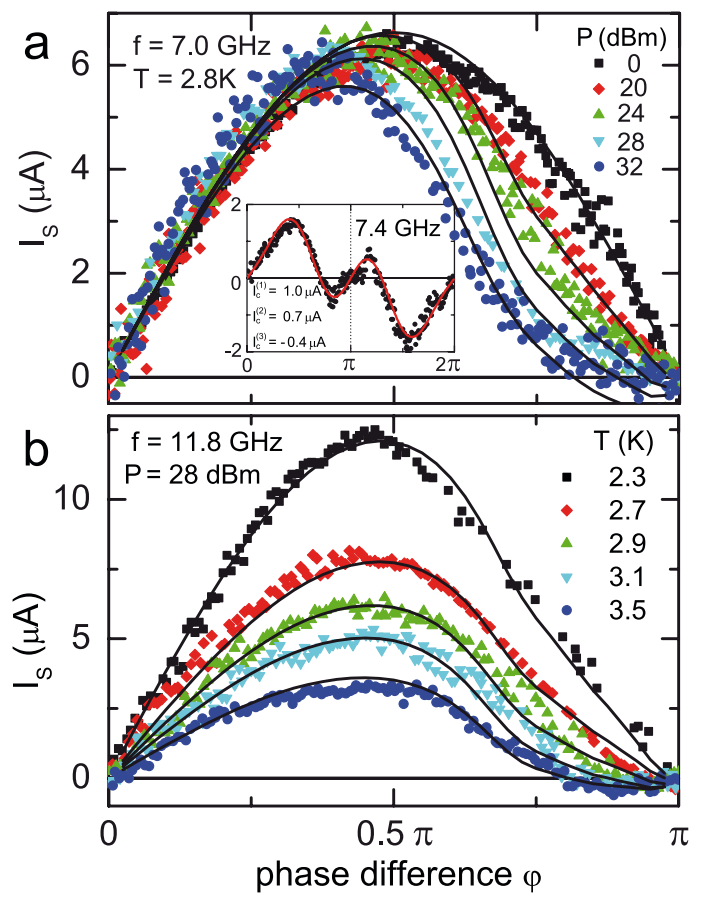

FIG. 3 (color online). (a) Measured CPR under irradiation with microwaves at $T=2.8 \mathrm{~K}$ and $f=7.0 \mathrm{GHz}$. (b) Measured CPR for different temperatures at $f=11.8 \mathrm{GHz}$. Solid lines represent the best fit to our model. Inset: Similar data at $7.4 \mathrm{GHz}$ (near a cavity resonance). The solid line is a Fourier expansion with the three coefficients listed. investigated at $P=28 \mathrm{dBm}$. The deviation from the sinusoidal form is moderate at $2.3 \mathrm{~K}$ and becomes more pronounced as $T$ is raised to $3.5 \mathrm{~K}$. Again the effects are most pronounced around $\varphi \simeq \pi$. At higher temperatures, the supercurrent becomes too small to be detected with our method. We have obtained qualitatively similar results on a shorter, but otherwise similar junction with $\Delta / \epsilon_{\mathrm{th}}=39$.

What is the origin of the microwave induced suppression of the supercurrent around $\varphi \simeq \pi$ ? The rf field is expected to excite small oscillations of the magnetic flux enclosed by the loop [17]. One possibility is that these oscillations lead to the induction of an ac voltage $V_{\mathrm{ac}}$ across the junction and modify the distribution function $f(\epsilon)$ of Andreev states within the junction. One would then expect sidebands in $f(\epsilon)$ of width $\hbar \omega_{\mathrm{rf}}$ [18]. Indeed, such a mechanism can lead to a distortion of the CPR qualitatively similar to our observations. However, it disagrees with our data in that the $I_{\mathrm{C}}^{(j)}(T)$ should again vanish exponentially for $j>1$ and $T \gtrsim \epsilon_{\mathrm{th}} / k_{B}$.

Another possibility is single-photon excitations of quasiparticles to ABS carrying supercurrent in the opposite direction. Such excitations become possible when the minigap goes to zero near $\varphi \simeq \pi$. To model this effect, we start, as in the equilibrium case, from Eq. (1), assuming that one can use the equilibrium supercurrent spectral density $\operatorname{Im} j_{\mathrm{S}}(\epsilon, \varphi)$ with a nonequilibrium distribution function $f(\epsilon)$.

A kinetic equation for $f(\epsilon)$ in dirty superconductors under microwave irraduation was formulated first by Eliashberg [19]. It can be represented in the form

$$
\begin{array}{r}
P_{\omega}[A(\epsilon)(f(\epsilon-\omega)-f(\epsilon))+B(\epsilon)(f(\epsilon+\omega)-f(\epsilon)) \\
+C(\epsilon)(1-f(\omega-\epsilon)-f(\epsilon))]=I[f(\epsilon)],
\end{array}
$$

where the coefficient $P_{\omega}=D\left|\delta \varphi_{\omega} / d_{\mathrm{eff}}\right|^{2}$ is proportional to the rf intensity. The phase oscillations $\delta \varphi_{\omega}$ represent the ac-response of the loop when excited by the microwave field. The coefficients $A(\epsilon), B(\epsilon)$, and $C(\epsilon)$ can be expressed through the Green's functions of the system [19]. We have approximated the Green's functions of our proximity superconductor by the standard BCS form and replaced $\Delta$ with the minigap $\epsilon_{g}(\varphi) . I[f(\epsilon)]$ is the scattering integral including electron-electron (ee) and electronphonon (ep) interactions. The first two terms $\propto P_{\omega}$ describe scattering of quasiparticles by photons and the third term pair-breaking, i.e., the creation of electron-hole pairs by single-photon absorption.

Assuming that the ee scattering is much stronger than the ep scattering, we approximate $f(\epsilon)$ by Fermi functions $f^{*}(\epsilon)$ with symmetrically shifted chemical potentials $\mu^{*}$ and $-\mu^{*}$ [10] for the microwave excited electron- and holelike quasiparticles, respectively. Since the excitation of quasiparticles by photons creates no charge imbalance, the nonequilibrium distribution function $f^{*}$ is particle-hole symmetric. Then $f^{*}$ and $\mu^{*}$ can be determined from Eq. (3) within a relaxation time approximation, rather than numerically solving the inhomogeneous kinetic equation. 

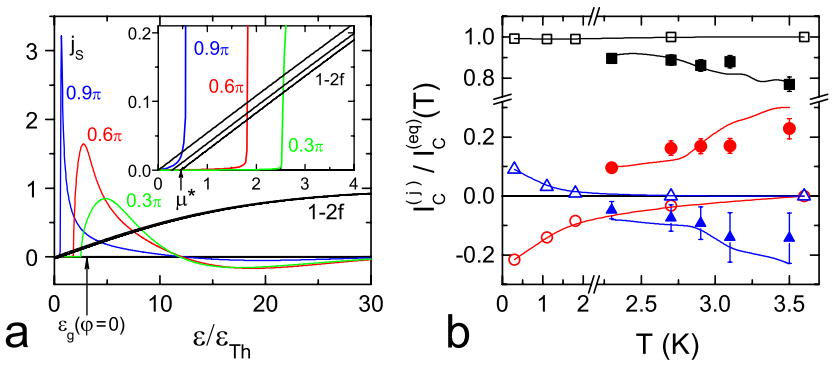

FIG. 4 (color online). (a) Spectral supercurrent $j_{\mathrm{S}}$ for $\varphi / \pi=$ $0.3,0.6$, and 0.9 together with $1-2 f^{*}$ vs $\epsilon$ at $2.8 \mathrm{~K}$. Inset: Zoom for $\epsilon / \epsilon_{\mathrm{th}} \leq 3$. Black lines: $1-2 f^{*}$ for $P=0,24$ and $32 \mathrm{dBm}$ (from top to bottom). The point $1-2 f=0$ defines the chemical potential $\mu^{*}$ for electronlike quasiparticles. (b) Normalized values of $I_{\mathrm{C}}^{(1)}(\boldsymbol{\square}, \square), I_{\mathrm{C}}^{(2)}(\bigcirc, \bigcirc)$, and $I_{\mathrm{C}}^{(3)}(\boldsymbol{\Delta}, \triangle)$ for the measured CPR without rf irradiation (open symbols) and for $11.8 \mathrm{GHz}$ and $P=28 \mathrm{dBm}$ (filled symbols) as a function of temperature. The lines are the theoretical curves corresponding to $f^{0}$ and $f^{*}$.

We neglect the $T$ dependence of the ep scattering time $\tau_{\text {ep }}$ in the limited $T$ interval under consideration. After integration of Eq. (3) over energy the terms containing $A(\epsilon)$ and $B(\epsilon)$ vanish, because they do not change the total number of excitations, and we obtain

$$
P_{\omega} \int_{0}^{\infty} C(\epsilon)\left(1-f^{*}(\omega-\epsilon)-f^{*}(\epsilon)\right) d \epsilon=\frac{\Delta n}{\tau_{\mathrm{ep}}},
$$

where $\Delta n=\int_{0}^{\infty}\left(f^{*}(\epsilon)-f^{0}(\epsilon)\right) d \epsilon$ and $f^{0}(\epsilon)$ is the equilibrium Fermi distribution function.

In Fig. 4(a) we plot $j_{\mathrm{S}}(\epsilon)$ together with $1-2 f^{*}(\epsilon)$. Despite the resulting small values $\mu^{*} \lesssim 0.5 \epsilon_{\text {th }}$, we have a noticeable effect on the total supercurrent $I_{\mathrm{S}}(\varphi)$ around $\varphi \simeq \pi$. The increase of $\mu^{*}$ cuts off the sharp positive peak of $j_{\mathrm{S}}$ at $\epsilon_{g}(\varphi)$ and enhances the relative weight of the negative part of $j_{\mathrm{S}}(\epsilon)$ at higher $\epsilon$ in Eq. (1). This can explain the observed sign reversal of $I_{\mathrm{S}}(\varphi)$. We express $P_{\omega} \tau_{\mathrm{ph}}=\eta(\omega) \exp (P[\mathrm{dBm}] / 10)$ in terms of a single parameter $\eta$, which also contains the $\omega$-dependent coupling between the sample loop and the antenna. $P$ is the externally applied microwave power. The results of our calculations are displayed as solid lines in Fig. 3 with $\eta(\omega)$ kept fixed to 0.001 for $f=7.0 \mathrm{GHz}$ and 0.005 for $11.8 \mathrm{GHz}$. All other parameters are identical to that of Fig. 2. The agreement achieved is quite satisfactory.

In Fig. 4(b) we show the Fourier coefficients of the measured CPR together with those extracted from the theory up to the 3rd harmonics. For a better comparison, the data are normalized with respect to the equilibrium critical current $I^{(\mathrm{eq})}$. In thermodynamic equilibrium (open symbols), the sign of the Fourier coefficients alternates and they decay monotonically with temperature. Under rf irradiation (full symbols), $I_{\mathrm{C}}^{(1)}$ slightly decreases with microwave power, which may result from slight electron heating by the microwaves. In contrast, $I_{\mathrm{C}}^{(2)}$ and $I_{\mathrm{C}}^{(3)}$ are much larger, increase with $T$, and have an opposite sign when compared to the case without rf irradiation. The experimental data agree rather well with the theoretical values. Our observations resemble very much the nonmonotonic temperature dependence of the amplitude of the subharmonic Shapiro steps observed in Refs. [5,6].

In conclusion, we have experimentally verified longstanding theoretical predictions on the current-phase relation in diffusive proximity Josephson junctions with highly transparent interfaces. We have presented strong evidence that microwave irradiation of the junction induces considerable anharmonicities in the current-phase relation. This effect can be understood in terms of a nonequilibrium occupation of Andreev bound states carrying the supercurrent through the junction. Our investigation calls for caution in the use of subharmonic Shapiro steps as a method for the determination of the current-phase relation.

We thank E. Scheer for support in instrumentation and T. Heikkilä and J.C. Cuevas for providing codes for a numerical evaluation of the spectral supercurrent and for stimulating discussions. This work has been supported by the DFG (STR 438-2 and GK 638).

[1] I. O. Kulik, Zh. Eksp. Teor. Fiz. 57, 1745 (1969) [Sov. Phys. JETP 30, 944 (1970)].

[2] For a review see, e.g., A. Golubov, M. Kupriyanov, and E. Il'ichev, Rev. Mod. Phys. 76, 411 (2004), and the references therein.

[3] T. T. Heikkilä, J. Särkkä, and F. K. Wilhelm, Phys. Rev. B 66, 184513 (2002).

[4] J. R. Waldram and J. M. Lumley, Rev. Phys. Appl. 10, 7 (1975).

[5] K. W. Lehnert et al., Phys. Rev. Lett. 82, 1265 (1999).

[6] P. Dubos, H. Courtois, O. Buisson, and B. Pannetier, Phys. Rev. Lett. 87, 206801 (2001).

[7] S. M. Frolov et al., Phys. Rev. B 70, 144505 (2004).

[8] A. K. Geim et al., Appl. Phys. Lett. 71, 2379 (1997).

[9] J. Sköldberg et al., Phys. Rev. Lett. 101, 087002 (2008); A. Zazunov et al., Phys. Rev. Lett. 90, 087003 (2003).

[10] Introduction to Superconductivity, edited by M. Tinkham (McGraw-Hill, New York, 1996), 2nd ed.

[11] P. Dubos et al., J. Vac. Sci. Technol. B 18, 122 (2000).

[12] M. Gaass et al., Phys. Rev. B 77, 024506 (2008).

[13] P. Dubos et al., Phys. Rev. B 63, 064502 (2001).

[14] Premature switching caused by thermal or quantum fluctuations can be neglected in our junctions.

[15] A. D. Zaikin and G. F. Zharkov, Fiz. Nizk. Temp. 7, 375 (1981) [Sov. J. Low Temp. Phys. 7, 184 (1981)].

[16] Neglecting the geometric inductance of the junction results in an underestimation of $I_{\mathrm{C}}$, which is less than $2 \%$ in our samples.

[17] These oscillations are strongly damped, because of the low $(\simeq 1 \Omega)$ quasiparticle resistance of the junctions.

[18] J. C. Cuevas (private communication).

[19] G. M. Eliashberg and B. I. Ivlev, in Nonequilibrium Superconductivity, edited by D. N. Langenberg and A. I. Larkin (North-Holland, Amsterdam, 1986), p. 211. 